\title{
The population of asteroids in the 2:1 mean motion resonance with Jupiter revised
}

\author{
Miroslav Brož ${ }^{1}$, D. Vokrouhlický ${ }^{1}$, F. Roig ${ }^{2}$, D. Nesvorný ${ }^{3}$, \\ W.F. Bottke $^{3}$ and A. Morbidelli ${ }^{4}$ \\ ${ }^{1}$ Institute of Astronomy, Charles University, V Holešovickách 2, 18000 Prague, Czech Republic \\ email: mira@sirrah.troja.mff.cuni.cz, vokrouhl@mbox.cesnet.cz \\ ${ }^{2}$ Observatório Nacional, Rua Gal. José Cristino 77, Rio de Janeiro, 20921-400 RJ, Brasil \\ email: froig@on.br \\ ${ }^{3}$ Department of Space Studies, Southwest Research Institute, 1050 Walnut St., Suite 400, \\ Boulder, CO 80302, USA \\ email: davidn@boulder.swri.edu, bottke@boulder.swri.edu \\ ${ }^{4}$ Observatoire de la Cǒte d'Azur, Dept. Cassiopee, BP 4224, 06304 Nice Cedex 4, France \\ email: alessandro.morbidelli@obs-nice.fr
}

\begin{abstract}
We study the population of asteroids inside the $2 / 1$ mean motion resonance with Jupiter, in the so called Hecuba gap. Origin of these bodies is not well understood: (i) the longlived (stable) population may be primordial, but this contradicts its steep size distribution, while (ii) the short-lived (unstable) population requires an efficient sustaining mechanism. Our working hypothesis is that the unstable asteroids are continuously resupplied from outside the resonance by the Yarkovsky effect. As a first step toward comparison of such model with observations, we report here an update of the observed population of asteroids residing in the 2/1 Jovian resonance, mainly because the number of cataloged orbits increased substantially during the last few years. We found there are 153 numbered and multi-opposition resonant asteroids in total and we classified them into the three sub-populations according to their dynamical lifetime. Our work also allowed us to derive several important parameters such as asteroid locations inside the resonance or size distribution of the sub-populations. As a particular novelty, we identified 6 asteroids located inside the high-eccentricity quasi-regular stable island, which previously seemed empty.
\end{abstract}

Keywords. Minor planets, asteroids

\section{Introduction and current state of art}

The structure of the Jovian mean motion resonances (MMRs) has been thoroughly studied for a long time. In the 1980's, there seemed to be a gap, almost void of any asteroids, in the $2 / 1 \mathrm{MMR}$, and that was in contrast with the $3 / 2 \mathrm{MMR}$, where numerous asteroids (so called Hildas) were observed. The 3-body models of motion in the 2/1 MMR (Murray 1986; Henrard \& Lemaître 1987; Lemaître \& Henrard 1990) failed to find any large chaotic regions inside, which could increase the eccentricity of the resonant objects and drive them to planet-crossing orbits, a preferred theory for the depletion of resonances since the work of Wisdom (1982). However, the early N-body numerical simulations (Wisdom 1987) showed the mechanism should work. Improved 3-body models (Morbidelli \& Moons 1993), with main secular perturbations of Jupiter's orbit, allowed to calculate borders of secular resonances embedded in the $2 / 1 \mathrm{MMR}$, but the key point was to use at least a 4-body model (Ferraz-Mello 1994, Henrard et al. 1995), because only then low- and high-eccentricity chaotic regions, caused by overlapping secondary or secular resonances, are interconnected and allow large scale diffusion. The extensive N-body simulations (Nesvorný \& Ferraz-Mello 1997) and semi-analytical analysis (Moons 
et al. 1998) allowed to map the structure of the $2 / 1 \mathrm{MMR}$ in detail. Two macroscopic islands of stability, separated by $\nu_{16}$ secular resonance, were denoted A and B (see also Fig. 4). In the 1990's, there were already 10 resonant asteroids discovered.

In general, observations support the latest theoretical results, confirming that the $2 / 1$ Jovian MMR harbors a small population of asteroids. Recent numerical analysis (Roig et al. 2002) allowed to classify these objects in three groups: (i) Zhongguos residing on stable orbits, whose dynamical lifetimes are comparable to the age of the Solar System, (ii) Griquas residing on orbits, whose dynamical lifetimes are several hundreds of My, and (iii) unstable asteroids, with a typical lifetime of $\simeq 10 \mathrm{My}$. Sub-populations (i) and (iii) are distinct not only by their very different lifetime, but also by their location in the resonance. Until now, no consistent scenario could make them linked together or to a common source.

Our goal is to prove that majority of the unstable asteroids have been pushed to the resonance from the main asteroid belt by the Yarkovsky effect. Before justifying any such hypothesis, we find useful to revise the $2 / 1 \mathrm{MMR}$ asteroid population anew, because the automated search programs significantly boosted the number of known asteroids and smart identification procedures allowed to efficiently link past observations with the new discoveries, making thus the orbits more accurate.

In this paper we report results of our new search of the $2 / 1$ resonant asteroids. The two main achievements are: (i) the population has grown to 153 objects with multiopposition orbits (thus nearly tripled during the last 3 years), and (ii) we found the first asteroids in the previously empty island A. Both results have interesting implications: more numerous populations allow to better characterize their size distributions - an information directly constraining their origin. In the same way, the numbers of asteroids in the stable islands A and B constrain mechanisms by which they have been injected in the resonance or later destabilized.

\section{The resonant population revised}

Selection procedure.- The primary sample of asteroids we used are the numbered and multi-opposition bodies from the AstOrb database of the Lowell observatory (ftp: //ftp. lowell.edu/pub/elgb/ as of April 2004) whose osculating semimajor axis $a$ and eccentricity $e$ satisfy $e>\frac{0.45}{0.14}\left(3.24-[a]_{\mathrm{AU}}\right)$ and $e>\frac{0.5}{0.12}\left([a]_{\mathrm{AU}}-3.34\right)$. This condition delimits a broad region near the $2 / 1$ MMR (see Fig. 1 in Roig et al. 2002) containing $\approx 4000$ asteroids. We numerically integrated their orbits for $10 \mathrm{ky}$ using SWIFT-MVs2-FP integrator. $\dagger$ Our simulation included 4 giant planets. We applied a barycentric correction to the initial conditions of the planets' and asteroids' orbits (to partially account for the indirect perturbation by terrestrial planets). The second-order symplectic integrator allows a longer time-step than SwIFT-MVs, in our case 91.3125 days.

For each of the orbits we computed the evolution of the critical angle $\sigma_{\mathrm{J} 2 / 1}=2 \lambda_{\mathrm{J}}-\lambda-\varpi$ that characterizes motion in the $2 / 1$ Jovian MMR $\left(\lambda_{\mathrm{J}}\right.$ and $\lambda$ are the longitude in orbit for Jupiter and for the asteroid, and $\varpi$ is the longitude of pericenter of the asteroid). The time-span of the integration was $10 \mathrm{ky}$ and the output sampling was $10 \mathrm{y}$. We computed two diagnostic parameters (Fig. 1): (i) the ratio $r_{\sigma}=\left(n_{+}-n_{-}\right) /\left(n_{+}+n_{-}\right)$, where $n_{+}$ (or $n_{-}$) is the number of data points for which $\sigma_{\mathrm{J} 2 / 1}(t)$ increased (or decreased) in two successive steps, and (ii) the difference $\Delta \sigma$ between the maximum and minimum value

$\dagger$ This is a modification of SwIFT package (Levison \& Duncan 1994) with the second-order symplectic integrator included (see Laskar \& Robutel 2001). Our program also contains on-line digital filters adapted to compute both synthetic proper orbital elements for the non-resonant motion and pseudo-proper elements for the resonant motion. 

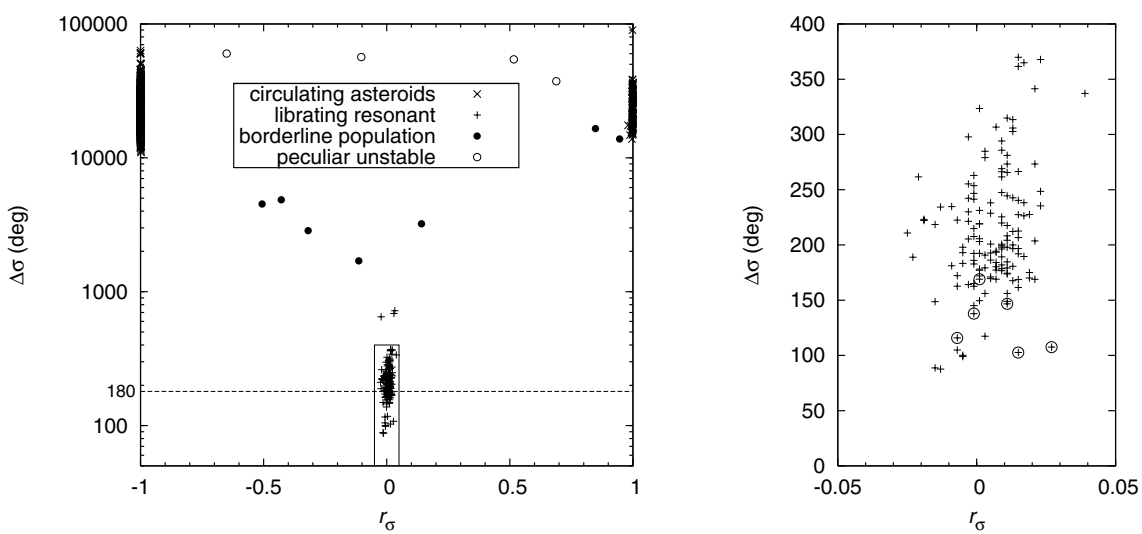

Figure 1. Left: The behavior of the critical angle $\sigma_{\mathrm{J} 2 / 1}$ of the $2 / 1 \mathrm{MMR}$ with Jupiter for asteroids in the broad region around and in the resonance. The total change $\Delta \sigma$ of $\sigma_{\mathrm{J} 2 / 1}$ (i.e. the maximum minus minimum value) vs. the ratio $r_{\sigma}$ (defined in the text) is plotted; both variables were computed from a 10 ky numerical integration with an output time-step $10 \mathrm{y}$. Bodies for which $\sigma_{\mathrm{J} 2 / 1}$ steadily circulates have either $r_{\sigma}=1$ or $r_{\sigma}=-1$; the four asteroids with $\Delta \sigma>30,000^{\circ}$ and $\left|r_{\sigma}\right|<0.98$ are either highly unstable or exhibit one abrupt change of $\sigma_{\mathrm{J} 2 / 1}(t)$ evolution. Asteroids with $r_{\sigma} \simeq 0$ and $\Delta \sigma \lesssim 360^{\circ}$ reside inside the resonance. Right: A zoom in the resonant population. Asteroids highlighted by circles were later identified to be located in the stable island A (see the text).

of $\sigma_{\mathrm{J} 2 / 1}$. Regularly librating resonant asteroids appear closely clustered near the point $\left(r_{\sigma}, \Delta \sigma\right)=\left(0,180^{\circ}\right)$ (in practice we used a $\left|r_{\sigma}\right|<0.05$ threshold). Asteroids outside the resonance, for which the critical angle circulates, have all $r_{\sigma} \doteq \pm 1$ and a value of $\Delta \sigma$ depending on the mean rate of their circulation. Since each of the parameters $r_{\sigma}, \Delta \sigma$ should allow by itself to detect the resonant asteroids, we can expect their values to be correlated - Fig. 1 confirms this conclusion. Several intermediate cases are: (i) borderline asteroids for which the critical angle alternates between periods of libration and circulation (full circles), and (ii) high-eccentricity asteroids with very unstable orbits or with peculiar orbits for which the sense of circulation of $\sigma_{\mathrm{J} 2 / 1}$ changes abruptly at some instant (open circles). Bodies with orbits for which $\sigma_{\mathrm{J} 2 / 1}$ librates, or temporarily librates, but which are non-resonant were excluded from Fig. 1. This is the case of motion near the pericentric and apocentric branches of periodic orbits in the three body problem. These orbits would have $\left|r_{\sigma}\right| \in(0.05,0.98)$ in our plot and also $N=\sqrt{a}\left(2-\sqrt{1-e^{2}} \cos I\right) \leqslant$ 0.8 ( $a$ is the osculating semimajor axis, $e$ the eccentricity and $I$ the inclination; see e.g. Morbidelli \& Moons 1993). There are many such pericentric alternators with $a \leqslant$ $3.275 \mathrm{AU}$ and $e \leqslant 0.15$, but we do not study them here.

The procedure described above led to us to identify 153 asteroids residing inside the 2/1 MMR with Jupiter, making the population significantly grown since the last analysis (Roig et al. 2002). The complete list of the resonant asteroids, which is too long to be published here, is available at http://sirrah.troja.mff.cuni.cz/yarko-site/.

Resonant asteroids: dynamical lifetime and classification into sub-populations.- Motion inside MMRs is chaotic enough that any single numerical integration of a chosen orbit may not necessarily represent the true future motion. A safer procedure is to consider (for each asteroid) a multitude of orbits which are close enough to be all compatible with the observations. $\dagger$ Hence, unlike the previous studies, we used

$\dagger$ In practice this means to consider orbits with slightly modified initial elements, well inside the uncertainty region of the orbit determination. We call such fictitious orbits, but otherwise statistically equivalent, "close clones". 


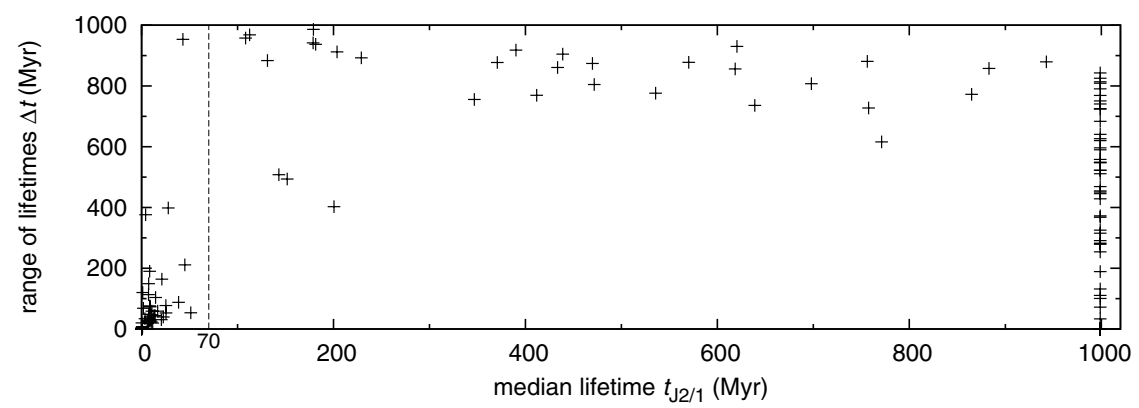

Figure 2. Median value $t_{\mathrm{J} 2 / 1}$ of lifetime vs. difference $\Delta t$ between the maximum and minimum lifetime values of the close clones for asteroids observed inside the 2/1 MMR with Jupiter. Asteroids with $t_{\mathrm{J} 2 / 1} \leqslant 70 \mathrm{My}$ have usually small $\Delta t$, indicating that all close clone orbits are unstable with a comparable timescale. Conversely, asteroids with $t_{\mathrm{J} 2 / 1} \geqslant 70 \mathrm{My}$ have large $\Delta t$, indicating some clones may be long-lived, while others are short-lived. This seems to distinguish Griqua-like orbits from the unstable orbits. Most Zhongguos (with $t_{\mathrm{J} 2 / 1}=1 \mathrm{~Gy}$ ) have again small or even zero value of $\Delta t$, since nearly all clones have the maximum lifetime.

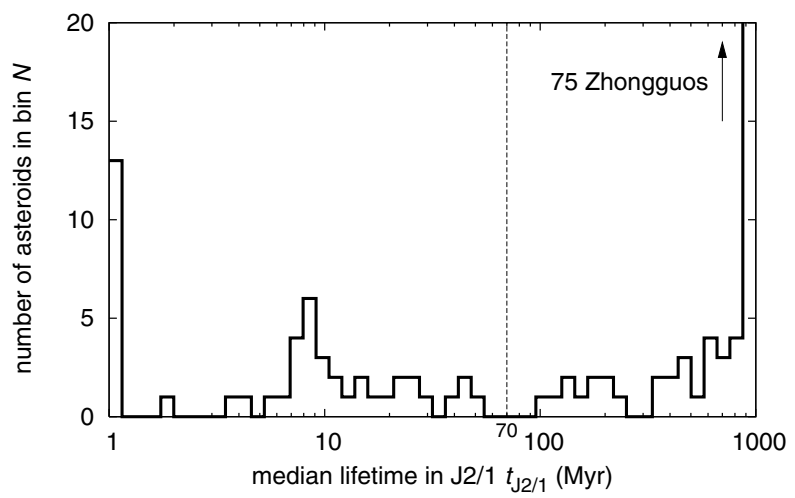

Figure 3. Distribution of the median lifetime values $t_{\mathrm{J} 2 / 1}$ for asteroids residing the $2 / 1 \mathrm{MMR}$. Note the log-binning and scale at the abscissa. Bodies with $t_{\mathrm{J} 2 / 1} \leqslant 70 \mathrm{My}$ seem to form a distinct population with unstable orbits; Zhongguos on stable orbits "pile up" at $t_{\mathrm{J} 2 / 1}=1 \mathrm{~Gy}$, limited in fact by the time-span of our integration. Griquas, with $t_{\mathrm{J} 2 / 1} \in(70 \mathrm{My}, 1 \mathrm{~Gy})$, seem to be adjacent to the Zhongguo group.

the close-clones technique to determine dynamical lifetimes of the resonant asteroids: for each body we generated 12 clones by changing the orbital semimajor axis and eccentricity by multiples of $\delta a=10^{-9} \mathrm{AU}$ and $\delta e=10^{-9}$; orbital inclination and angular elements of clones were the same as of the original orbit. We numerically integrated resulting $13 \times 153$ orbits using SwIFT-MVs2-FP integrator upto 1 Gy or until the filtered mean semimajor axes (i.e. with fast oscillations removed) showed a significant departure (larger than $0.05 \mathrm{AU}$ ) from the centre of the resonance at $3.27 \mathrm{AU}$. This procedure was found reliable enough to characterize the residence time inside the resonance. Since all close clones are statistically equivalent realizations of the orbit, the characteristic dynamical lifetime $t_{\mathrm{J} 2 / 1}$ of the corresponding asteroid was determined as a median value of the individual lifetimes of the clones.

Figures 2 and 3 summarize the result. The first, showing aside the median lifetime $t_{\mathrm{J} 2 / 1}$ also the range $\Delta t$ of the individual close-clone lifetime values, suggests there is a transition at about $t_{\mathrm{J} 2 / 1} \simeq 70 \mathrm{My}$ : (i) asteroids with smaller values of $t_{\mathrm{J} 2 / 1}$ reside on unstable orbits so that almost none of the close clones happen to have a long dynamical lifetime; (ii) asteroids with larger values of $t_{\mathrm{J} 2 / 1}$ reside on marginally stable or 

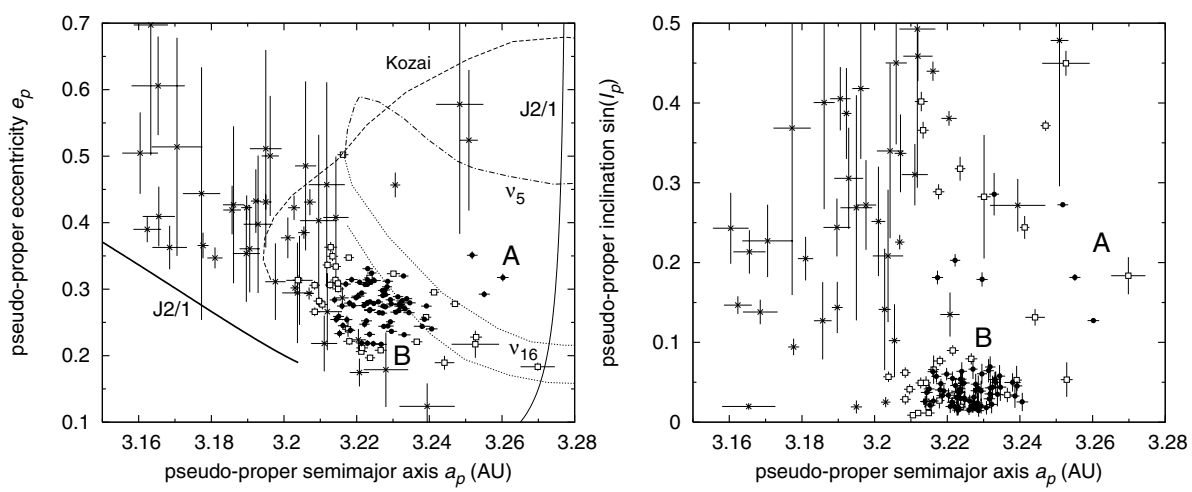

Figure 4. Distribution of the resonant asteroids in the space of pseudo-proper elements $a_{p}, e_{p}$, $\sin I_{p}$. Zhongguos are denoted by filled circles, Griquas by open squares and the unstable asteroids by crosses. The error bars denote standard deviation of pseudo-proper elements computed over $1 \mathrm{My}$ interval of time. The thin and thick solid lines in the $\left(a_{p}, e_{p}\right)$ projection (left) are the libration centre and separatrix of the $2 / 1$ resonance (both shown for $I=0^{\circ}$ ). Borders of chaotic zones associated with embedded secular resonances $-\nu_{5}, \nu_{16}$ and the Kozai resonance - are the dashed and dashed-dotted lines (all shown for $I=10^{\circ}$; adapted from Moons et al. 1998). A majority of the stable asteroids is clustered in the island B, while a few of them (see Table 1) are located in the island A, characterized by a higher mean eccentricity and inclination. All unstable asteroids are located in the chaotic zone where various secular resonances overlap. Griquas are a border-line population mostly at the edge of the B-region. In fact, 2 -D projections shown here always lack clarity in showing 3-D structures; for that reason we posted a 3-D animation of the resonance structure with positions of the embedded asteroids at our web-site http:// sirrah.troja.mff.cuni.cz/yarko-site/.

stable orbits, characterized with some (or all) clones having very long dynamical lifetimes (eventually $\geqslant 1$ Gy). Additionally, Fig. 3 suggests bodies with $t_{\mathrm{J} 2 / 1}<70 \mathrm{My}$ are perhaps composed of two distinct groups: (i) very short-lived with $t_{\mathrm{J} 2 / 1} \leqslant 1 \mathrm{My}$, and (ii) shortlived with $t_{\mathrm{J} 2 / 1} \geqslant 2 \mathrm{My}$. The very short-lived group ( $\simeq 25 \%$ of the unstable asteroids) might be populated from a different source than the adjacent main belt, such as the Jupiter family comets or near-Earth asteroids, but this issue needs to be studied with care before drawing any conclusions. The long-lived asteroids $\left(t_{\mathrm{J} 2 / 1} \geqslant 70 \mathrm{My}\right)$ are either marginally stable Griquas or stable Zhongguos. $\dagger$ The respective numbers of asteroids are: 47 unstable asteroids, 31 Griquas and 75 Zhongguos.

Resonant asteroids: location inside the resonance.- Our numerical integration allowed us to characterize also the location of asteroids inside the $2 / 1 \mathrm{MMR}$. This was done by computing so called pseudo-proper (resonant) orbital elements (e.g. Roig et al. (2002)) - semimajor axis $a_{p}$, eccentricity $e_{p}$ and inclination $I_{p}$. They are defined as osculating orbital elements at the time when the orbit intersects a surface of section defined by $\sigma_{\mathrm{J} 2 / 1}=0^{\circ}$ and $\dot{\sigma}_{\mathrm{J} 2 / 1}<0$ and $\varpi-\varpi_{\mathrm{J}}=0^{\circ}$ and $\Omega-\Omega_{\mathrm{J}}=0^{\circ}$, where $\left(\Omega, \varpi ; \Omega_{\mathrm{J}}, \varpi_{\mathrm{J}}\right)$ are the longitude of node and pericenter of the asteroid and of Jupiter, respectively, and $\dot{\sigma}_{\mathrm{J} 2 / 1}$ is the time derivative of $\sigma_{\mathrm{J} 2 / 1}$. In practice, though, we used a weakened condition $\sigma_{\mathrm{J} 2 / 1} \leqslant$ $5^{\circ}$ and $\Delta \sigma<0$ and $\left|\varpi-\varpi_{\mathrm{J}}\right| \leqslant 5^{\circ}$ (where $\Delta \sigma$ is a difference between the two successive values of $\sigma_{\mathrm{J} 2 / 1}$ ), in accord with Roig et al. (2002). The pseudo-proper resonant elements are near constants of motion in the averaged three-body problem with Jupiter on a fixed circular orbit; in reality the motion of both the asteroids and planets is more complicated and this may cause variations of $\left(a_{p}, e_{p}, I_{p}\right)$. We thus computed mean values of $\left(a_{p}, e_{p}, I_{p}\right)$

$\dagger$ In fact, our 1 Gy simulation is still inappropriate to quantify lifetimes of Zhongguos, it only indicates they are longer than $1 \mathrm{~Gy}$. Griquas are separated from Zhongguos only conventionally and up to this time-span they seem to form a shorter-lived "tail" of the Zhongguo population. 

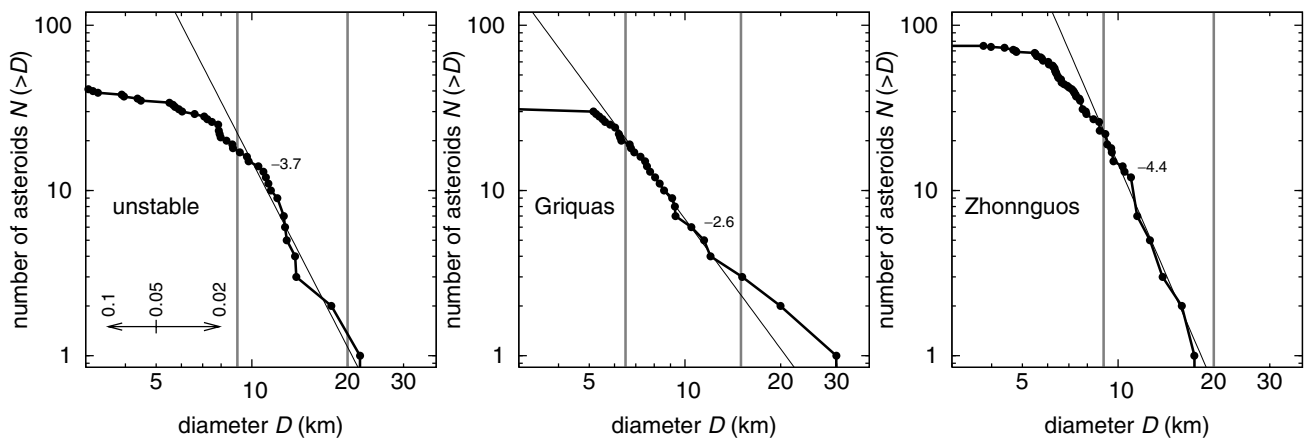

Figure 5. Cumulative size-frequency distributions of highly unstable asteroids (left), Griquas (middle) and Zhongguos (right). The solid lines indicate the best fit power-law approximations within the size interval delimited by the vertical grey lines. There are statistically significant differences in steepness of the size distributions of the three sub-populations. We assumed the albedo of all asteroids to be 0.05 . Arrows in the left-most plot indicate a horizontal displacement of the distributions, if we assume different values of the albedo -0.1 and 0.02 .

and standard deviations $\left(\sigma_{a}, \sigma_{e}, \sigma_{I}\right)$ during the first 1 My of our integration (only nominal orbits were used for that purpose). Results are shown in Fig. 4, where we give positions of the resonant asteroids projected onto the planes $\left(a_{p}, e_{p}\right)$ and $\left(a_{p}, \sin I_{p}\right)$. We note there is a strong correlation between the lifetimes of objects and their positions in the resonance: (i) the unstable orbits are located inside the chaotic regions where secular resonances overlap, (ii) the stable orbits (Zhongguo-like) are inside the stable islands B and A, and (iii) the marginally stable (Griqua-like) are right at the outskirts of the stable regions.

Resonant asteroids: size distribution.- Having been able to classify resonant asteroids into the three sub-groups we are now ready to study some of their parameters indicative of their origin or evolution. An important information is a size-frequency distribution (SFD) shown in Fig. 5. We assumed the value of albedo to be 0.05 , appropriate for the prevalent $\mathrm{C}$ - and D-type asteroids in the outer part of the main asteroid belt; higher or smaller mean albedo values would displace the distributions toward smaller or larger sizes as shown in the left panel. The largest members of each of the sub-populations are of about the same size $(\simeq 20 \mathrm{~km})$. We fitted SFDs with a power-law $N(>D) \sim D^{\alpha}$, usually down to the diameter $\simeq 9 \mathrm{~km}$, that does not seem to be significantly affected by the observational incompleteness. We found the following values of the SFD exponent $\alpha$ : (i) $-4.4 \pm 0.3$ for Zhongguos, (ii) $-2.6 \pm 0.5$ for Griquas, and (iii) $-3.7 \pm 0.2$ for the unstable asteroids. $\dagger$ In spite of relatively large standard deviations of the exponents, the SFDs differ from each other with a high statistical significance. The SFDs of Zhongguos and the unstable asteroids are also significantly steeper than -2.5 , what one would expect for a collisionally evolved isolated system (e.g. Dohnanyi 1969). Even if generalizations of the original Dohnanyi's model are taken into account, such as a size-dependence of the disruption $Q$-factor (e.g. O'Brien \& Greenberg 2003), the SFD of the unstable asteroids is hard to reconcile with a system evolved solely by collisions. It is also interesting to compare the SFDs of the resonant asteroids, the unstable asteroids in particular, with that of a large population of asteroids adjacent to the 2/1 MMR. Within a comparable interval of sizes $(9-50 \mathrm{~km}$, say) we found that the cumulative SFD of the main belt population is well approximated by a power-law with an exponent $-2.60 \pm 0.05$.

$\dagger$ We warn the results for the long-lived populations - Zhongguos and Griquas - are partly ambiguous, because these populations are not well separated. The current division at $t_{\mathrm{J} 2 / 1}=1 \mathrm{~Gy}$ was chosen a little arbitrarily. If the whole long-lived population (i.e. with $t_{\mathrm{J} 2 / 1} \geqslant 70 \mathrm{My}$ ) is considered together its SFD has an exponent $-3.5 \pm 0.2$. 
Table 1. Parameters of Zhongguo- and Griqua-like asteroids found to reside in the stable island A of the 2/1 MMR with Jupiter. $a_{p}, e_{p}$ and $I_{p}$ are pseudo-proper semimajor axis, eccentricity and inclination, $\sigma_{a}, \sigma_{e}$ and $\sigma_{I}$ their standard deviations during a $1 \mathrm{My}$ numerical integration, $t_{\mathrm{J} 2 / 1}$ median lifetime, $H$ absolute magnitude and $D$ diameter, calculated from $H$ using the value of albedo 0.05. The last column is the classification: $\mathrm{G}$ - Griquas, Z - Zhongguos. Asteroid (4177) Kohman is a border-line case (see the text for discussion).

\begin{tabular}{llrrrrrrrrrr}
\hline No. Name & $\begin{array}{c}a_{p} \\
{[\mathrm{AU}]}\end{array}$ & $e_{p}$ & $\begin{array}{c}I_{p} \\
{[\mathrm{deg}]}\end{array}$ & $\begin{array}{c}\sigma_{a} \\
{[\mathrm{AU}]}\end{array}$ & $\sigma_{e}$ & $\begin{array}{c}\sigma_{I} \\
{[\mathrm{deg}]}\end{array}$ & $\begin{array}{c}t_{\mathrm{J} 2 / 1} \\
{[\mathrm{My}]}\end{array}$ & $\begin{array}{c}H \\
{[\mathrm{mag}]}\end{array}$ & $\begin{array}{c}D \\
{[\mathrm{~km}]}\end{array}$ & Class. \\
\hline 78801 & $2003 \mathrm{AK}_{88}$ & 3.260 & 0.318 & 7.309 & 0.002 & 0.006 & 0.14 & 1000 & 15.20 & 5.50 & $\mathrm{Z}$ \\
& $1999 \mathrm{VU}_{218}$ & 3.241 & 0.295 & 14.125 & 0.001 & 0.002 & 0.82 & 771 & 15.25 & 5.37 & $\mathrm{G}$ \\
& $2001 \mathrm{FY}_{84}$ & 3.253 & 0.217 & 26.727 & 0.007 & 0.020 & 0.89 & 152 & 14.06 & 9.29 & $\mathrm{G}$ \\
& $2003 \mathrm{SA}_{197}$ & 3.252 & 0.351 & 15.807 & 0.001 & 0.006 & 0.09 & 1000 & 14.63 & 7.15 & $\mathrm{Z}$ \\
& $2003 \mathrm{YN}_{94}$ & 3.255 & 0.293 & 10.451 & 0.002 & 0.005 & 0.24 & 1000 & 15.20 & 5.50 & $\mathrm{Z}$ \\
& $2004 \mathrm{FG}_{32}$ & 3.247 & 0.278 & 21.816 & 0.001 & 0.004 & 0.37 & 535 & 14.53 & 7.48 & $\mathrm{G}$ \\
\hline \multirow{2}{*}{4177} & Kohman & 3.233 & 0.320 & 16.598 & 0.001 & 0.001 & 1.52 & 1000 & 12.70 & 17.38 & $\mathrm{Z}$ \\
\hline
\end{tabular}

This is interesting because its difference from the SFD exponent of the unstable asteroids is approximately $-1 . \dagger$ This supports models in which the unstable asteroids are resupplied with a size-selective process which strength is inversely proportional to size. The Yarkovsky effect (e.g. Vokrouhlický et al., this volume) meets this constraint (see also Morbidelli \& Vokrouhlický 2003). These models, however, cannot be extended to Zhongguos, because of their anomalously steep SFD.

Resonant asteroids: objects inside the stable island A.- Among the newly identified asteroids inside the 2/1 MMR with Jupiter we found 6 cases that lie above the separatrix of the $\nu_{16}$ secular resonance (see Table 1 and Fig. 4). These are the first known objects in the previously void stable island A. In comparison to B-Zhongguos, A-Zhongguos (and also A-Griquas) have typically smaller $\left(<90^{\circ}\right)$ amplitude of $\sigma_{\mathrm{J} 2 / 1}(t)$ (see Fig. 1). There is also a clear secular term with an approximate amplitude of $20^{\circ}$ and period $\simeq 9 \mathrm{ky}$. The sizes of objects, excluding Kohman, range from $5.5 \mathrm{~km}$ to $7.5 \mathrm{~km}$, if we adopt the nominal geometric albedo 0.05. The ratio of the number of A-Zhongguos (excluding A-Griquas) and the number of $\mathrm{B}$-Zhongguos is equal to $3 / 71 \doteq 0.04$. However, this ratio has only a low statistical significance, because the number of A-Zhongguos, discovered upto now, is too small and new discoveries will change the ratio a lot.

Models that would attempt to explain the origin and evolution of the stable population in the 2/1 MMR should consider the differences between island A and island B population as important constraints. For instance, Ferraz-Mello et al. (1998) discussed a possibility, that Zhongguos are relics of a primordial population. They proved the island A would become significantly more (but not completely) depleted if the mutual position of Jupiter and Saturn were such that the period of Great Inequality in Jupiter's motion would be shorter. (This is a plausible scenario since the giant planets likely migrated during the early evolution of the Solar system.) On the other hand, the steep SFD of Zhongguos (Fig. 5) would indicate a recent collisional origin of a majority of this population. However, the mean values of both eccentricity and inclination for A-Zhongguos and B-Zhongguos differ so much (the ejection velocities would be of order $1 \mathrm{~km} / \mathrm{s}$ ), that a possibility of a collisional break-up taking part in the island B and populating with fragments the island A is very unlikely.

$\dagger$ In fact, when we discard the extremely unstable asteroids with $t_{\mathrm{J} 1 / 2} \leqslant 1 \mathrm{My}$ (see Fig. 3 ), which may have anomalous origin, the size distribution of the remaining unstable asteroids becomes slightly shallower and the difference from the main belt population is $\simeq-0.8$. 
We finally note that the asteroid (4177) Kohman, previously classified as Griqua-like by Roig et al. (2002), is a border-line case residing very close to the separatrix of the $\nu_{16}$ resonance. Indeed, we found the critical angle of this resonance $\left(\sigma_{16}=\Omega-\Omega_{\mathrm{S}}\right)$ exhibits periods of librations and circulations in Kohman's case. $\dagger$ With its large size $(17 \mathrm{~km}$ in diameter) this body would by far dominate the island A population.

\section{Conclusions}

We revised the currently observed population of asteroids residing in the $2 / 1$ mean motion resonance with Jupiter. We identified 153 resonant asteroids, and among them six new located in the stable island A. The properties of the resonant population will serve as important constraints for models of its origin and evolution (e.g. for the model involving the Yarkovsky effect to push main belt asteroids onto unstable resonant orbits). To make such models even more constrained, the following further observations would be particularly useful: (i) accurate astrometry of faint asteroids to complete the known population down to smaller sizes, with the goal to reduce uncertainty in determination of the power-law representation of the size-frequency distribution; (ii) spectral or multicolor observations to investigate similarity or diversity of the resonant sub-populations and of possible sources, as far as the surface mineralogy is concerned; (iii) lightcurve observations of the unstable asteroids with the goal to obtain (or at least constrain) their pole positions. $\ddagger$ However, spectral and lightcurve observations of a large number of small main-belt asteroids will probably not be available until we have data from space-born projects (such as GAIA).

\section{Acknowledgements}

We thank the referee, Sylvio Ferraz-Mello, for valuable comments and discussion. The work of MB and DV was supported by the Grant Agency of the Czech Republic.

\section{References}

Dohnanyi, J.W. 1969, J. Geophys. Res. 74, 2531

Ferraz-Mello, S. 1994, in Asteroids, Comets, Meteors 1993, eds. A. Milani et al., Kluwer Academic Publishers, Dordrecht, 1994, p. 175

Ferraz-Mello, S., Michtchenko, T.A. \& Roig, F. 1998, Astron. J. 116, 1491

Henrard, J. \& Lemaître, A. 1987, Icarus 69, 266

Henrard, J., Watanabe, N. \& Moons, M. 1995, Icarus 115, 336

Laskar, J. \& Robutel, P. 2001, Celest. Mech. Dyn. Astr. 80, 39

Lemaître, A. \& Henrard, J. 1990, Icarus 83, 391

Levison, H.F. \& Duncan, M.J. 1994, Icarus 108, 18

Moons, M., Morbidelli, A. \& Migliorini, F. 1998, Icarus 135, 458

Morbidelli, A. \& Moons, M. 1993, Icarus 102, 316

Morbidelli, A. \& Vokrouhlický, D. 2003, Icarus 163, 120

Murray, C.A. 1986, Icarus 65, 70

Nesvorný, D. \& Ferraz-Mello, S. 1997, Icarus 130, 247

O'Brien, D.P. \& Greenberg, R. 2003, Icarus 164, 334

Roig, F., Nesvorný, D. \& Ferraz-Mello, S. 2002, Mon. Not. R. Astron. Soc. 335, 417

Wisdom, J. 1982, Astron. J. 87, 577

Wisdom, J. 1987, Icarus 72, 241

$\dagger$ The close proximity of $\nu_{16}$ resonance leads to a relatively larger value of $\sigma_{I}$ for this body; similarly, the asteroid $1999 \mathrm{VU}_{218}$ is near the resonance, though in this case the critical angle circulates.

$\ddagger$ Note the Yarkovsky-driven origin of the unstable population would, for instance, predict most of these asteroids should have prograde rotation. 